Ambiente \& Água - An Interdisciplinary Journal of Applied Science
ISSN 1980-993X - doi:10.4136/1980-993X
www.ambi-agua.net
E-mail: ambi.agua@gmail.com

\title{
Influência do número de classes de vulnerabilidade na determinação da suscetibilidade morfométrica à inundação
}

\author{
doi:10.4136/ambi-agua.1842 \\ Received: 16 Jan. 2016; Accepted: 15 May 2016 \\ Vívian Gemiliano Pinto ${ }^{1 *}$; Ricardo Neves de Souza Lima ${ }^{2}$; \\ Ricardo Costa Pinto e Santos ${ }^{1}$; Celso Bandeira de Melo Ribeiro ${ }^{3}$ \\ ${ }^{1}$ Instituto Federal de Educação Ciência e Tecnologia do Sudeste de Minas Gerais (IFSudesteMG), \\ Juiz de Fora, MG, Brasil \\ Departamento de Educação e Tecnologia \\ ${ }^{2}$ Fundação Instituto Brasileiro de Geografia e Estatística (IBGE), Rio de Janeiro, RJ, Brasil \\ Centro de Documentação e Disseminação de Informações (CDDI) \\ ${ }^{3}$ Universidade Federal de Juiz de Fora (UFJF), Juiz de Fora, MG, Brasil \\ Departamento de Engenharia Sanitária e Ambiental (ESA/UFJF) \\ *Autor correspondente: e-mail: viviangemiliano@gmail.com, \\ riksou@yahoo.com.br, ricardo.cpsantos@gmail.com, celso.bandeira@ufff.edu.br
}

\section{RESUMO}

A morfometria da bacia hidrográfica é um importante instrumento de diagnóstico da suscetibilidade à inundação. Este diagnóstico pode nortear o planejamento e a implementação de medidas mitigadoras para se evitar prejuízos causados por alagamentos. O objetivo deste trabalho foi estudar a suscetibilidade à inundação da bacia hidrográfica do Ribeirão do Espírito Santo (BHRES), localizada no município de Juiz de Fora, Minas Gerais, Brasil, por meio de sua caracterização morfométrica utilizando-se duas, três e cinco classes de vulnerabilidade. Para isso a bacia foi subdividida considerando dois níveis de detalhamento: no primeiro, menos detalhado, foram geradas três sub-bacias e no segundo, mais detalhado, foram geradas 65 microbacias. Nestas unidades de estudo foi avaliada a suscetibilidade morfométrica à inundação e os resultados foram comparados utilizando-se as técnicas de agrupamento "K-means e Fuzzy C-means". Os resultados demonstraram que o número de classes de vulnerabilidade adotada influencia o resultado da classificação das áreas, sugerindo que a utilização de critérios de validação de cluster pode ser usada para balizar tal escolha. Agrupamentos formados apenas por semelhanças morfométricas se distinguem daqueles obtidos pela metodologia utilizada para classificação de áreas suscetíveis à inundação, visto que a metodologia para análise de suscetibilidade transforma os valores numéricos de cada parâmetro morfométrico em uma classe de suscetibilidade, ponderando-o conforme sua implicação na inundação.

Palavras-chave: microbacias, morfometria, ribeirão do Espírito Santo, técnicas de agrupamento.

\section{Influence of the number of vulnerability classes in determining morphometric susceptibility to flooding}

\begin{abstract}
Watershed morphometry is an important tool to diagnose susceptibility to flooding. This diagnosis can guide the planning and implementation of mitigation measures to avoid
\end{abstract}


flooding damage. The objective of this work was to study flooding susceptibility in Espírito Santo Stream Basin (ESSB), located in the municipality of Juiz de Fora, Minas Gerais, Brazil, based on its morphometric characterization using two, three and five classes of vulnerability. In order to accomplish this, the basin was divided considering two levels of detail: in the first, less-detailed level, three sub-basins were generated; and in the second, more-detailed level, 65 watersheds were generated. Morphometric susceptibility to flooding was evaluated in these study units and the results were compared using the K-means and Fuzzy C-means clustering techniques. The results showed that the number of classes adopted affects the result of classification vulnerability, suggesting that cluster validation criteria can be used to delimit the choice of the number of classes to be adopted. Groups formed only by morphometric similarities differ from those obtained by the methodology based on classification of areas susceptible to flooding, since the methodology for susceptibility analysis transforms the numerical values of each morphometric parameter in a class of susceptibility.

Keywords: clustering techniques, Espírito Santo stream, morphometry, watershed.

\section{INTRODUÇÃO}

A Lei $n^{\circ}$ 9.433, de 8 de janeiro de 1997 que institui a Política Nacional de Recursos Hídricos considera a bacia hidrográfica como unidade de gestão dos recursos hídricos. Entretanto, desde os anos 1980, os estados do Paraná ("Paraná Rural") e Santa Catarina ("Microbacias") já possuíam alguns programas de manejo e conservação dos recursos naturais tendo como foco a escala de microbacias (Neves Neto e Hespanhol, 2009). Prática defendida por vários autores, pois as microbacias são as grandes alimentadoras dos rios e dos grandes sistemas fluviais (Machado e Stipp, 2003; Lima, 2010; Machado, 2012).

Contudo os termos sub-bacia e microbacia hidrográfica apesar de incorporados na literatura técnico-científica, não apresentam a convergência conceitual observada para o termo bacia hidrográfica (Teodoro et al., 2007). Neste estudo adotou-se como sub-bacias, as áreas de drenagem dos tributários do curso d'água principal e como microbacias as áreas formadas por canais de $2^{\mathrm{a}}, 3^{\mathrm{a}}$ e, em alguns casos, de $4^{\mathrm{a}}$ ordem.

Num contexto de gestão de bacias e microbacias, a morfometria fornece ferramentas para mensuração e análise matemática da configuração da superfície terrestre e da forma e dimensões de sua paisagem (Cooke e Doornkamp, 1974). Devido à estreita correspondência entre as características morfométricas das bacias hidrográficas e o comportamento hidrológico, aquelas corroboram para o entendimento do regime hidrológico (Fritzsons et al., 2009).

Entretanto, a grande variabilidade espacial das características físicas dos componentes do ciclo hidrológico, em sua fase terrestre, e a complexidade na geração de informações de variáveis morfométricas evidencia a premência pela utilização das ferramentas de Sistemas de Informações Geográficas (SIG), enquanto, bancos de dados espaciais e técnicas de sensoriamento remoto vêm apoiar o entendimento da dinâmica espacial e temporal na manipulação de informações especializadas em sistemas de bacias hidrográficas.

Verifica-se, ainda, que a morfometria da microbacia hidrográfica é um importante instrumento de diagnóstico da susceptibilidade à inundação, e que seus resultados podem nortear o planejamento e a implementação de medidas mitigadoras para evitar os prejuízos causados pelos alagamentos (Rodrigues e Starzynski, 2003).

Contudo, inquere-se se as análises de vulnerabilidade, tão comuns em diagnósticos ambientais podem ser influenciadas pela quantidade de classes definidas a priori. Sendo assim, o objetivo deste trabalho foi determinar a suscetibilidade morfométrica à inundação de sub-bacias e microbacias da bacia hidrográfica do Ribeirão do Espírito Santo (BHRES) em 
Juiz de Fora, Minas Gerais, Brasil, adotando-se duas, três e cinco classes de vulnerabilidade. Além disso, aplicaram-se diferentes métodos de agrupamento para identificar semelhanças morfométricas entre microbacias e compararam-se os grupos formados pelas classes de suscetibilidade à inundação com aqueles obtidos analisando-se apenas as semelhanças entre as microbacias.

\section{MATERIAL E MÉTODOS}

\section{1. Área de estudo}

A área de estudo compreende a BHRES, que tem como curso d’água principal o ribeirão Espírito Santo, afluente da margem direita do Rio Paraibuna, que, por sua vez, é afluente da margem esquerda do Rio Paraíba do Sul. A BHRES está localizada na região noroeste do município de Juiz de Fora, Minas Gerais, Brasil (Figura 1), com uma área de drenagem de $151,49 \mathrm{~km}^{2}$, predominância de alta susceptibilidade à erosão, apesar de sua vocação rural, diferenciando-se apenas em áreas próximas ao exutório, onde se observam granjeamentos, grandes indústrias, incluindo parte do distrito industrial da cidade, uma estação de tratamento de água e uma estação de tratamento de esgoto (Pinto et al., 2014).

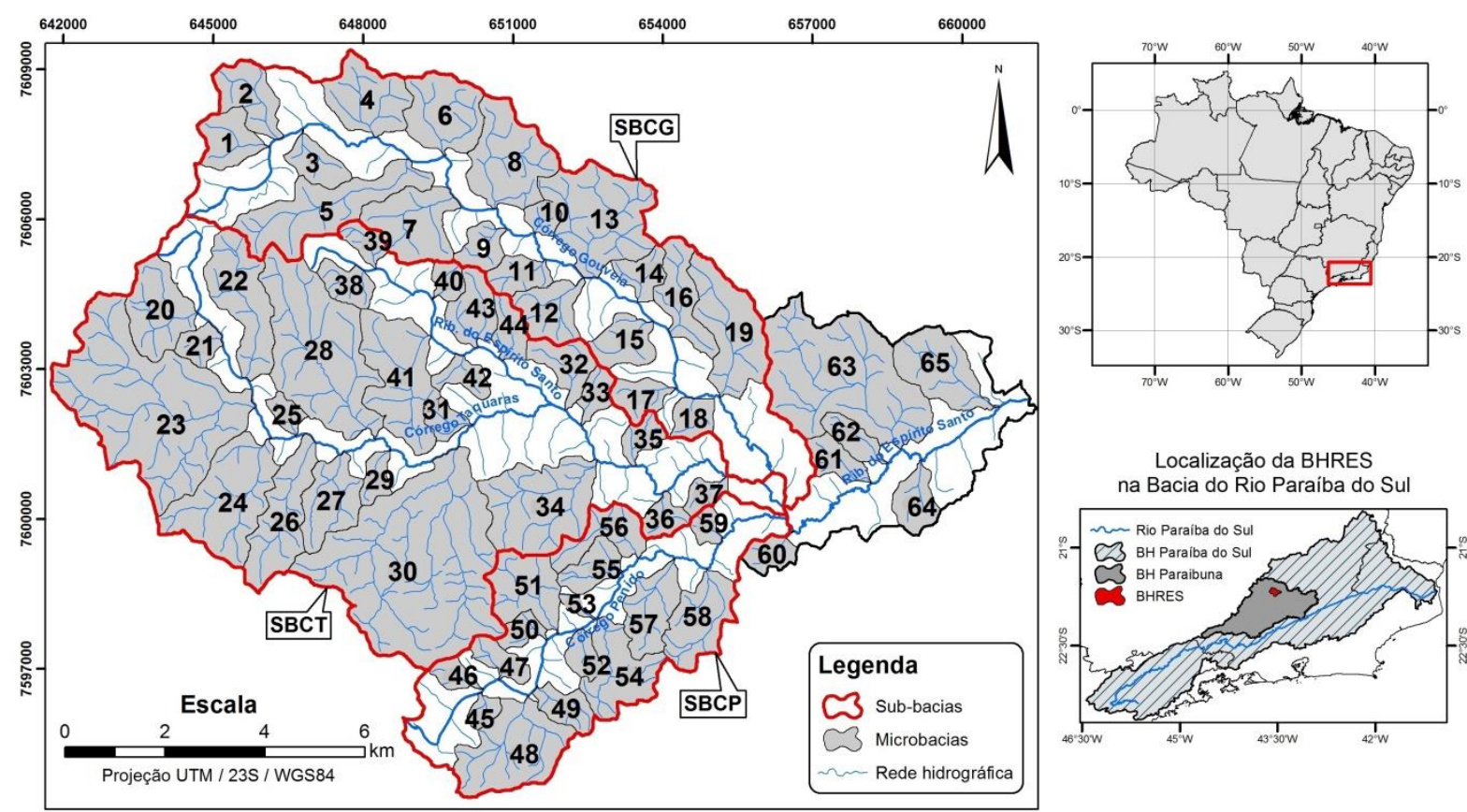

\begin{tabular}{|c|c|c|c|c|c|c|c|c|c|c|c|c|c|c|c|c|c|c|c|c|c|}
\hline Cod. & Microbacia & Cod. & \begin{tabular}{|l|} 
Microbacia \\
\end{tabular} & Cod. & Microbacia & Cod. & \begin{tabular}{|l|} 
Microbacia \\
\end{tabular} & Cod. & Microbacia & Cod. & Microbacia & Cod. & \begin{tabular}{|l|} 
Microbacia \\
\end{tabular} & \begin{tabular}{|l|} 
Cod. \\
\end{tabular} & Microbacia & Cod. & Microbacia & Cod. & Microbacia & Cod. & Microbacia \\
\hline 1 & $01 \mathrm{GE}$ & 7 & $07 G D$ & 13 & $13 \mathrm{GE}$ & \begin{tabular}{|l|}
19 \\
\end{tabular} & $19 \mathrm{GE}$ & 25 & \begin{tabular}{|l|}
$06 \mathrm{TE}$ \\
\end{tabular} & 31 & $12 \mathrm{TE}$ & 37 & $18 \mathrm{TD}$ & 43 & 06 ESE & 49 & $05 P D$ & 55 & $11 \mathrm{PE}$ & 61 & 02 JUSE \\
\hline 2 & $02 \mathrm{GE}$ & 8 & $08 \mathrm{GE}$ & 14 & $14 \mathrm{GE}$ & 20 & $01 \mathrm{TD}$ & 26 & $07 T D$ & 32 & $13 \mathrm{TE}$ & 38 & $01 \mathrm{ESD}$ & 44 & $07 \mathrm{ESE}$ & 50 & $06 \mathrm{PE}$ & 56 & $12 \mathrm{PE}$ & 62 & 03 JUSE \\
\hline 3 & $03 G D$ & 9 & 09 G D & 15 & $15 G D$ & 21 & 02 TD & 27 & $08 \mathrm{TD}$ & 33 & $14 \mathrm{TE}$ & 39 & $02 \mathrm{ESE}$ & 45 & $01 P D$ & 51 & $07 \mathrm{PE}$ & 57 & $13 P D$ & 63 & 04 JUS E \\
\hline 4 & $04 \mathrm{GE}$ & 10 & $10 \mathrm{GE}$ & 16 & $16 \mathrm{GE}$ & 22 & $03 \mathrm{TE}$ & 28 & $09 \mathrm{TE}$ & 34 & $15 \mathrm{TD}$ & 40 & $03 \mathrm{ESE}$ & 46 & $02 \mathrm{PE}$ & 52 & $08 P D$ & 58 & $14 \mathrm{PD}$ & 64 & 05 JUS D \\
\hline 5 & 05 GD & 11 & $11 G D$ & 17 & $17 G D$ & 23 & 04 TD & 29 & $10 \mathrm{TD}$ & 35 & $16 \mathrm{TE}$ & 41 & 04 ES D & 47 & $03 \mathrm{PE}$ & 53 & $09 \mathrm{PE}$ & 59 & $15 \mathrm{PE}$ & 65 & 06 JUSE \\
\hline 6 & $06 \mathrm{GE}$ & 12 & $12 \mathrm{GD}$ & 18 & $18 \mathrm{GD}$ & 24 & $05 \mathrm{TD}$ & 30 & $11 \mathrm{TD}$ & 36 & $17 T D$ & 42 & $05 \mathrm{ESD}$ & 48 & $04 \mathrm{PD}$ & 54 & $10 P D$ & 60 & 01 JUS D & & \\
\hline
\end{tabular}

Figura 1. Localização da bacia hidrográfica do ribeirão do Espírito Santo, Juiz de Fora, Minas Gerais e distribuição de suas três sub-bacias e 65 microbacias.

\subsection{Morfometria da BHRES}

Para a caracterização morfométrica da BHRES foi gerado no SIG, ArcGIS 10, um modelo digital de elevação hidrograficamente condicionado (MDEHC), a partir de levantamento por LIDAR (Light Detection and Ranging) e restituição aerofotogramétrica da bacia realizados pela prefeitura Municipal de Juiz de Fora. Para a identificação dos corpos d'água foram utilizados dados vetoriais digitais referentes à hidrografia das cartas 
topográficas do Instituto Brasileiro de Geografia e Estatística (IBGE), folhas Ewbank da Câmara (SF-23-X-C-VI-2) e Juiz de Fora (SF-23-X-D-IV-1), ambas na escala de 1:50.000 (IBGE, 1980b; 1980a).

Para a determinação da morfometria da bacia, além de considerá-la como um todo, esta também foi dividida em três sub-bacias principais (Figura 1): Sub-bacia do Córrego Gouveia (SBCG); Sub-bacia do Córrego Taquaras (SBCT), que contém a nascente do Ribeirão Espírito Santo; e Sub-bacia do Córrego Penido (SBCP).

Além da divisão em sub-bacias, a BHRES também foi subdividida em 65 microbacias. As microbacias foram delimitadas a partir dos afluentes de segunda ordem dos principais cursos d’água da BHRES, que são: Córrego Gouveia, Córrego Penido, Córrego Taquaras e Ribeirão do Espírito Santo. Cabe ressaltar que, apesar do Ribeirão do Espírito Santo emprestar o nome à BHRES, o Córrego Taquaras possui maior extensão a montante que o Ribeirão Espírito Santo, ao se unir a este (Figura 1) (Pinto et al., 2014).

A delimitação das áreas foi realizada automaticamente no módulo "Spatial Analyst" do SIG ArcGIS a partir do MDEHC, sobre o qual havia sido determinado em SIG a direção do escoamento superficial pelo método desenvolvido por Jenson e Domingue (1988).

Os parâmetros morfométricos utilizados para a caracterização das unidades hidrográficas, objetivando identificar áreas mais suscetíveis a inundações são apresentados na Tabela 1.

\subsection{Definição das faixas de suscetibilidade à inundação na BHRES}

Para identificar as áreas mais suscetíveis a inundação na BHRES, avaliou-se a bacia e suas principais sub-bacias (Figura 1).

Inicialmente, atribuiu-se valor 5 para a condição mais favorável à inundação e valor 1 a condição menos favorável. Dividindo-se o intervalo entre os valores extremos em cinco classes obteve-se uma escala de suscetibilidade variando de 1 a 5 , correspondendo, respectivamente, à: muito baixa, baixa, média, alta e muito alta suscetibilidade à inundação.

Após a determinação da suscetibilidade para cada um dos parâmetros morfométricos, estes foram somados tanto para a BHRES quanto para cada sub-bacia. Como se utilizou 12 parâmetros, cuja escala variava de um a cinco, o somatório para cada unidade de estudo variou de 12 a 60. Sendo assim, estabeleceram-se cinco novos intervalos de domínio de classe: entre 12 e 21,6; maior que 21,6 e 31,2; maior que 31,2 e 40,8; maior que 40,8 e 50,4 e; maior que 50,4 e 60, correspondendo, respectivamente, à suscetibilidade à inundação muito baixa, baixa, média, alta e muito alta, identificando-se assim, a suscetibilidade da BHRES e de suas principais sub-bacias.

A fim de observar se ocorreria variação na classificação realizada conforme a quantidade de intervalos de classe criados. Aplicou-se a mesma metodologia para três e duas classes, adotando-se, respectivamente, a classificação alta, média e baixa e; alta e baixa suscetibilidade.

\subsection{Avaliação da suscetibilidade à inundação das microbacias pertencentes à BHRES}

Posteriormente, com a finalidade de precisar dentro da BHRES as áreas com características morfométricas mais vulneráveis à inundação, esta foi subdividida em 65 microbacias e os 12 parâmetros, descritos na Tabela 1, foram calculados para cada uma delas. Para cada parâmetro foram determinados o valor médio entre as microbacias e o desvio padrão. Todas as microbacias que possuíam valor de parâmetro fora do intervalo: média mais ou menos duas vezes o desvio padrão, foram considerados valores anômalos, e receberam valor máximo ou mínimo de acordo com a implicação do parâmetro na suscetibilidade à inundação. Por exemplo, uma microbacia que possuísse declividade média do canal principal (Dmcp) acima do intervalo considerado aceitável, receberia classificação 1, uma vez que altos valores de Dmcp são desfavoráveis à inundação. Em contrapartida, valores extremamente elevados de densidade hidrográfica (Dh) são favoráveis a inundação e receberiam 


\section{classificação 5 .}

Feito isto, aplicou-se a mesma metodologia de identificação de áreas mais suscetíveis à inundação, descrita para a BHRES e suas sub-bacias utilizando-se cinco, três e duas classes de suscetibilidade.

Tabela 1. Descrição dos parâmetros morfométricos utilizados na área de estudo.

\begin{tabular}{|c|c|}
\hline \multicolumn{2}{|r|}{ Características geométricas da bacia } \\
\hline Parâmetro & Descrição \\
\hline Área de Drenagem (A) & Área em projeção horizontal delimitada por seus divisores topográficos $\left(\mathrm{km}^{2}\right)$. \\
\hline Perímetro $(\mathrm{P})$ & Comprimento da linha divisora de águas que limita a bacia hidrográfica $(\mathrm{km})$. \\
\hline Fator de Forma $(\mathrm{F})$ & $\begin{array}{l}\text { É a razão ente a área de drenagem }\left(\mathrm{km}^{2}\right) \text { e quadrado do comprimento do eixo da bacia } \\
(\mathrm{km}) \text {. Uma bacia com } \mathrm{F} \text { baixo indica que a mesma é menos susceptível a enchentes } \\
\text { que outra, de mesmo tamanho, porém com fator de forma maior (Villela e Mattos, } \\
\text { 1975). }\end{array}$ \\
\hline Índice de Circularidade (IC) & $\begin{array}{l}\text { É função da razão entre a área de drenagem }\left(\mathrm{km}^{2}\right) \text { e o quadrado do perímetro }(\mathrm{km}) \text {. } \\
\text { Bacias com IC maiores que } 0,51 \text { indicam tendência a formas circulares, com maior } \\
\text { susceptibilidade a enchente, enquanto valores inferiores a } 0,51 \text { indicam uma tendência } \\
\text { à formas alongadas que favorecem a translação e maior tempo de armazenamento do } \\
\text { fluxo nos canais (Alves e Castro, 2003). }\end{array}$ \\
\hline Coeficiente de Compacidade (Kc) & $\begin{array}{l}\text { É função da razão entre o perímetro }(\mathrm{km}) \text { e a raiz da área de drenagem }\left(\mathrm{km}^{2}\right) \text {. } \\
\text { Quanto maior o valor deste coeficiente mais irregular é a forma da bacia, sendo que } \\
\text { valores próximos da unidade correspondem a bacias circulares, enquanto acima de } \\
1,47 \text { indicam bacias com forma alongada (Villela e Mattos, 1975). }\end{array}$ \\
\hline \multicolumn{2}{|r|}{ Características da rede de drenagem } \\
\hline Parâmetro & Descrição \\
\hline
\end{tabular}

Ordem Hierárquica da Bacia $(\mathrm{O})$

Consiste na classificação de determinado curso d’água conforme o número de tributários que recebe (Christofoletti, 1981). Neste trabalho foi utilizada a classificação proposta por Strahler (1952). Indica o grau de ramificação da bacia, mantendo relação direta com a área da bacia, descarga e capacidade de escoamento. Sendo que a descarga aumenta exponencialmente em função da ordem hierárquica do canal (Horton, 1945).

Frequência de Canais de Primeira Ordem $(\mathrm{Fc})$

Declividade Média do Canal Principal (Dmcp)

Densidade de Drenagem (Dd)

Densidade Hidrográfica (Dh)

Densidade de Confluência (Dc)

Índice de Sinuosidade do Canal Principal (Is)
Frequência de canais que não possuem tributários, considerando a classificação proposta por Strahler (1952) em relação ao número total de canais. Um elevado número de canais de primeira ordem está relacionado a um rápido fluxo de água para fora da bacia, indicando mais água sendo escoada do que infiltrada (Morisawa, 1962).

É a razão entre a amplitude altimétrica do curso d água principal (m) e o comprimento do canal principal (m). É um fator importante na formação do escoamento superficial e consequentemente na modelagem e evolução da rede de drenagem, implicando nos processos de inundação/enchente (Zãvoianu, 1985).

É a razão entre o comprimento total de todos os canais $(\mathrm{km})$ e a área de drenagem total $\left(\mathrm{km}^{2}\right)($ Horton, 1945). É controlada por inúmeras variáveis: relevo, cobertura vegetal, volume de chuvas, infiltração de água no solo, resistência à erosão entre outras (Souza, 2005).

É a relação existente entre o número de canais e a área da bacia hidrográfica $\left(\mathrm{km}^{2}\right)$ (Horton, 1945). Indica a resposta da bacia ao processo de escoamento superficial, seu comportamento hidrográfico e a capacidade de geração de novos cursos d’água (Souza, 2005).

É a razão entre o número total de confluências de canais e à área total da bacia $\left(\mathrm{km}^{2}\right)$. É um parâmetro diretamente relacionado à capacidade de escoamento das águas (Zãvoianu, 1985).

É a razão entre o comprimento do rio principal $(\mathrm{km})$ e a distância vetorial entre os pontos extremos do talvegue $(\mathrm{km})$. Is igual a um, revela que o canal de drenagem tende a ser retilíneo, superior a dois, canal tortuoso e valores intermediários indicam formas transicionais(Alves e Castro, 2003).

\section{IPABH}




\subsection{Identificação da similaridade morfométrica das microbacias}

A identificação da similaridade morfométrica entre as microbacias foi obtida por meio de análise de agrupamento, que é uma técnica multivariada amplamente utilizada em diversas áreas do conhecimento.

Uma questão importante em análise de agrupamento é a definição do número de grupos a serem divididos. Para tanto, procedeu-se a validação dos grupos, ou seja, submeteram-se os dados morfométricos das microbacias a alguns testes para identificar o número ideal de grupos. No caso, como não havia informação a priori sobre a forma de uma solução de agrupamento esperada ou conhecida, utilizou-se os seguintes critérios de validação: PBM (Pakhira et al., 2004), VPC (Dave, 1996) e Xie-Beni (1991), que foram implementados no programa Matlab (The Mathworks Inc., 2012).

Neste estudo utilizaram-se duas técnicas de agrupamento: "K-means" e "Fuzzy Cmeans" (FCM) para agrupar as microbacias conforme suas características morfométricas. O método "K-means" é um algoritmo de agrupamento rígido enquanto o FCM gera um padrão probabilístico de pertinência (Singh et al., 2011).

$\mathrm{O}$ "K-means" é um dos algoritmos de agrupamento mais utilizados mundialmente devido à sua simplicidade e eficiência computacional (Singh et al., 2011). Enquanto, a técnica "Fuzzy" permite estimar ou atribuir participações parciais de qualquer área estudada para um conjunto definido por uma classe morfométrica, consequentemente, representar melhor a continuidade espacial de superfícies de terreno. Ela tem a vantagem de permitir que uma microbacia possa ser membro de mais de um conjunto de características morfométricas, e que a definição desses conjuntos (classes) não precisam ser mutuamente excludentes (Wang et al., 2010).

O algoritmo FCM utilizado foi desenvolvido por Santos (2006) e implementado no programa Matlab (The Mathworks Inc., 2012). E o agrupamento pelo método "K-means" foi obtido utilizando o programa Statística 6.0 (Statsoft Inc., 2002).

\section{RESULTADOS E DISCUSSÃO}

\subsection{Morfometria da BHRES e sub-bacias}

A BHRES possui altitude máxima de $952,5 \mathrm{~m}$, dentro da SBCG, e altitude mínima de 678,4 $\mathrm{m}$ próxima ao exutório. A altitude média da BHRES é de 771,7 $\mathrm{m}$, sendo que a subbacia com maior altitude média é a SBCT $(781,8 \mathrm{~m})$, seguida pela SBCG $(776,3 \mathrm{~m})$ e SBCP (759 m).

A BHRES possui padrão de drenagem dendrítico, com grande quantidade de afluentes e subafluentes. A maior sub-bacia da BHRES é a SBCT, com área de drenagem de $68,7 \mathrm{~km}^{2}$, correspondendo a 45,35\% da área da bacia; seguida da SBCG, com área de $42,73 \mathrm{~km}^{2}(28,2 \%$ da BHRES); SBCP, com área de 22,71 $\mathrm{km}^{2}$ (14,99\% da BHRES); totalizando 88,54\% da BHRES.

Tanto a BHRES, quanto suas sub-bacias apresentam características geométricas de baixa suscetibilidade à inundação, com fator de forma variando entre 0,20 e 0,39 , índice de circularidade entre 0,18 e 0,24 e índice de compacidade entre 2,01 e 2,35, indicando formas alongadas segundo Villela e Mattos (1975). Sendo que os índices mais desfavoráveis à inundação pertencem a SBCG e os mais favoráveis pertencentes à BHRES.

Quanto às características da rede de drenagem, a BHRES é de $6^{\mathrm{a}}$ ordem, as SBCT e SBCG de $5^{\mathrm{a}}$ ordem e a SBCP de $4^{\mathrm{a}}$ ordem. O rio principal da BHRES possui $28,93 \mathrm{~km}$, abarcando o rio principal da SBCT que mede $20,8 \mathrm{~km}$ (Figura 1), enquanto os rios principais da SBCG e SBCP medem respectivamente, 20 e 12,2 km. A altitude máxima do talvegue na BHRES é 946,82 m e a mínima 678,44 m, com amplitude altimétrica de 268,38 m.

A BHRES e suas sub-bacias possuem alta capacidade de drenagem de acordo com a 
classificação proposta por Beltrame (1994), com densidades de drenagem próximas a três. A sub-bacia com maior densidade de drenagem, densidade hidrográfica e densidade de confluências é a SBCP $\left(3,08 \mathrm{~km}^{-1}, 5,11 \mathrm{~km}^{-2}, 3,70 \mathrm{~km}^{-2}\right)$, seguida da SBCT $\left(2,93 \mathrm{~km}^{-1}\right.$, $\left.4,15 \mathrm{~km}^{-2}, 3,16 \mathrm{~km}^{-2}\right)$ e SBCG $\left(2,63 \mathrm{~km}^{-1}, 3,83 \mathrm{~km}^{-2}, 2,74 \mathrm{~km}^{-2}\right)$. A densidade de drenagem, densidade hidrográfica e densidade de confluências da BHRES, como um todo, são respectivamente, $2,83 \mathrm{~km}^{-1}, 4,09 \mathrm{~km}^{-2}$ e $3,07 \mathrm{~km}^{-2}$.

Os índices de sinuosidade da BHRES $(1,63)$, da SBCT $(1,54)$ e SBCG $(1,5)$ são iguais ou superiores a 1,5, o que segundo Christofoletti (1981) caracteriza os canais como meandrosos. Apenas o índice de sinuosidade da SBCP é 1,41, ligeiramente inferior.

A BHRES apresentou características geométricas desfavoráveis à inundação, porém as características da rede de drenagem indicaram certa vulnerabilidade. Ao aplicar a metodologia de análise de suscetibilidade à inundação, independente do número de classes adotado para classificar a BHRES, esta se apresentou como altamente suscetível à inundação, se comparada com suas sub-bacias. Contudo, as sub-bacias tiveram a categorização variável conforme o número de classes adotado. Os resultados sinalizam que a SBCG é a menos suscetível à inundação seguida da SBCP e a mais suscetível entre as sub-bacias é a SBCT.

\subsection{Suscetibilidade à inundação na BHRES e sub-bacias}

A sub-bacia que se mostrou menos suscetível a inundação foi a SBCG, que foi classificada como de muito baixa vulnerabilidade, quando foram adotadas cinco classes, e de baixa vulnerabilidade quando se adotaram duas e três classes. A SBCP foi considerada de média suscetibilidade quando se adotaram três e cinco classes, mas foi classificada como de baixa suscetibilidade ao se adotar apenas duas classes. Enquanto a SBCT, apesar de receber a classificação de média suscetibilidade ao se adotarem três e cinco classes, ela passou a ser considerada de alta suscetibilidade na classificação utilizando-se duas classes (Figura 2). Já a BHRES foi classificada como de alta vulnerabilidade independente do número de classes adotadas.

\subsection{Morfometria das microbacias}

Entre as 65 microbacias contidas na BHRES, 19 delas estão localizadas na SBCG, 25 na SBCT e 15 estão localizadas na SBCP, havendo ainda seis microbacias localizadas na área à jusante das três sub-bacias.

A maior microbacia é a 11TD, localizada na margem direita do Córrego Taquaras com $10,68 \mathrm{~km}^{2}$ e perímetro de $20,11 \mathrm{~km}$, enquanto a menor microbacia é a 09PE, localizada na margem esquerda do Córrego Penido com $0,14 \mathrm{~km}^{2}$ de área e perímetro de $2,55 \mathrm{~km}$. A média das áreas das microbacias é $1,65 \mathrm{~km}^{2}$, com desvio padrão de $1,89 \mathrm{~km}^{2}$.

A menor microbacia (09PE) apresenta a maior densidade de drenagem $\left(8,31 \mathrm{~km}^{-1}\right)$, densidade hidrográfica $\left(21,25 \mathrm{~km}^{-2}\right)$, densidade de confluência $\left(7,05 \mathrm{~km}^{-2}\right)$ e declividade média do canal principal $\left(0,16 \mathrm{~m} \cdot \mathrm{m}^{-1}\right)$.

A menor densidade de drenagem $\left(2,06 \mathrm{~km}^{-1}\right)$, densidade hidrográfica $\left(2,76 \mathrm{~km}^{-2}\right)$ e densidade de confluência $\left(0,92 \mathrm{~km}^{-2}\right)$ são identificadas na microbacia $01 \mathrm{GE}$ que possui $1,09 \mathrm{~km}^{2}$ de área.

A microbacia 05GD apresenta o menor fator de forma $(0,17)$, menor índice de circularidade $(0,18)$ e maior coeficiente de compacidade $(2,32)$, enquanto a microbacia $10 \mathrm{GE}$ apresenta maior fator de forma $(0,61)$, maior índice de circularidade $(0,63)$ e menor coeficiente de compacidade $(1,36)$. A média e desvio padrão para o fator de forma, índice de circularidade e coeficiente de compacidade são, respectivamente, 0,$39 ; 0,09 ; 0,36 ; 0,07 ; 1,69$; 0,17 .

Entre as 65 microbacias, 44 são de $2^{\mathrm{a}}$ ordem, 18 de $3^{\mathrm{a}}$ ordem e apenas três de $4^{\mathrm{a}}$ ordem, uma na SBCG (13GE) e duas na SBCT (04TD e 11TD), sendo a microbacia 04TD a que 
apresenta menor declividade média do canal principal $\left(0,02 \mathrm{~m} \cdot \mathrm{m}^{-1}\right)$. Com relação à frequência de canais de primeira ordem $(\mathrm{Fc})$, as microbacias 05GD e 07TD apresentam as maiores frequências $(89 \%)$, enquanto a microbacia 07PE apresenta a menor frequência (64\%). A média de Fc é $73 \%$ e o desvio padrão 6,7\%.

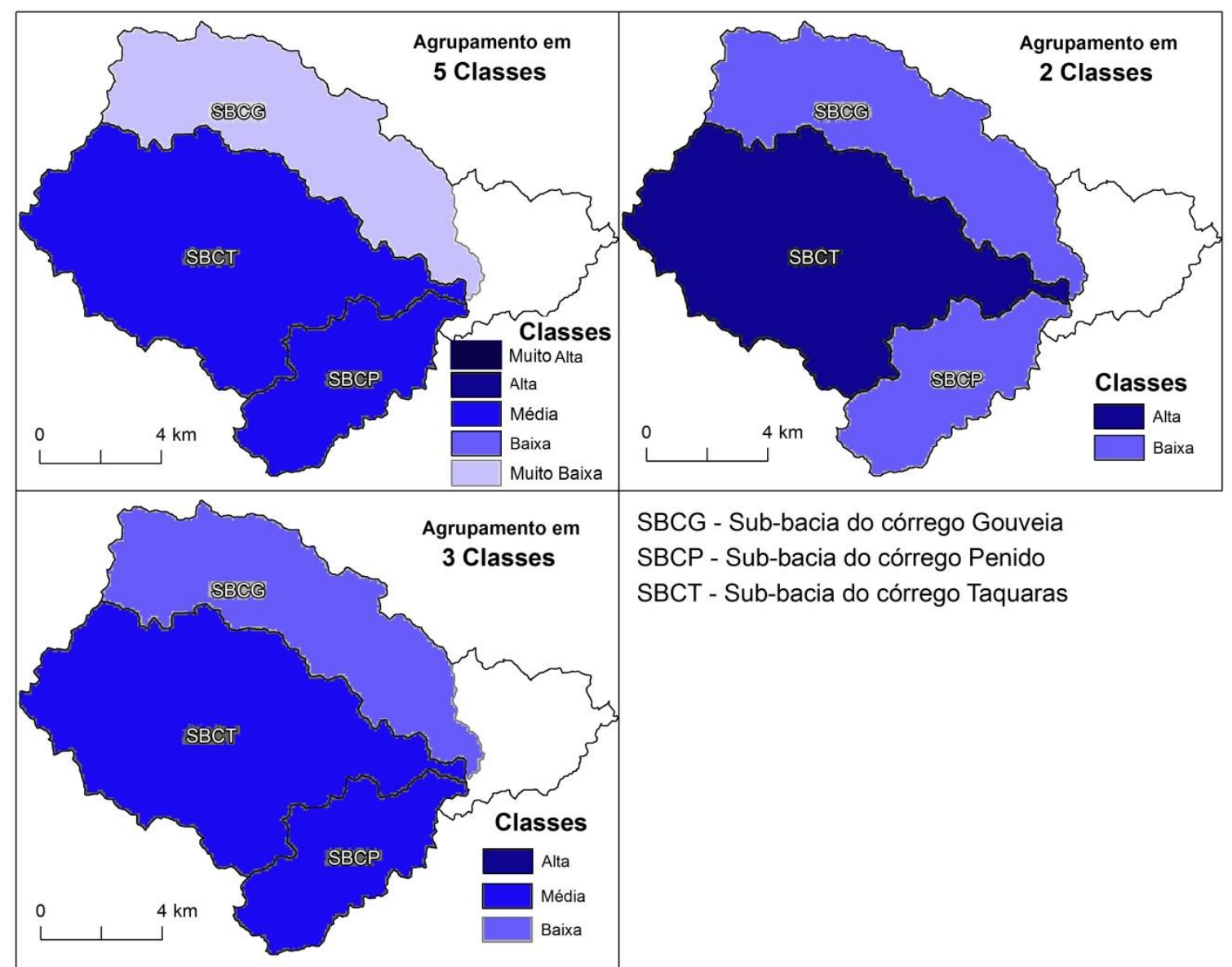

Figura 2. Suscetibilidade à inundação nas sub-bacias da BHRES, classificando-as em duas, três e cinco classes de vulnerabilidade.

\subsection{Suscetibilidade à inundação das microbacias pertencentes à BHRES}

A partir dos dados morfométricos calculados para os 12 parâmetros, apresentados na Tabela 1, classificou-se todos os parâmetros morfométricos conforme descrito na metodologia.

Para a classificação, utilizando-se apenas duas classes, 25 microbacias foram classificadas como de alta e 40 como de baixa suscetibilidade a inundação. Na SBCG, 14 microbacias foram classificadas como de baixa e cinco como de alta; na SBCT, 13 microbacias foram classificadas como de baixa e 12 de alta; na SBCP, oito de baixa e sete de alta e; na área de drenagem à jusante das sub-bacias, cinco foram classificadas como de baixa e uma como de alta (Figura 3).

Utilizando-se três classes de vulnerabilidade, 18 microbacias foram classificadas como de baixa, 43 como de média e quatro, como de alta vulnerabilidade. Na SBCG, cinco microbacias foram classificadas como de baixa, 12 como de média e duas como de alta (08GE e $13 \mathrm{GE}$ ); na SBCT, cinco foram classificadas como de baixa, 18 como de média e duas como de alta (11TD e 15TD); na SBCP, cinco foram classificadas como de baixa e dez como de média; na área de drenagem à jusante das sub-bacias, três foram classificadas como baixa e três como média suscetibilidade à inundação (Figura 3). 


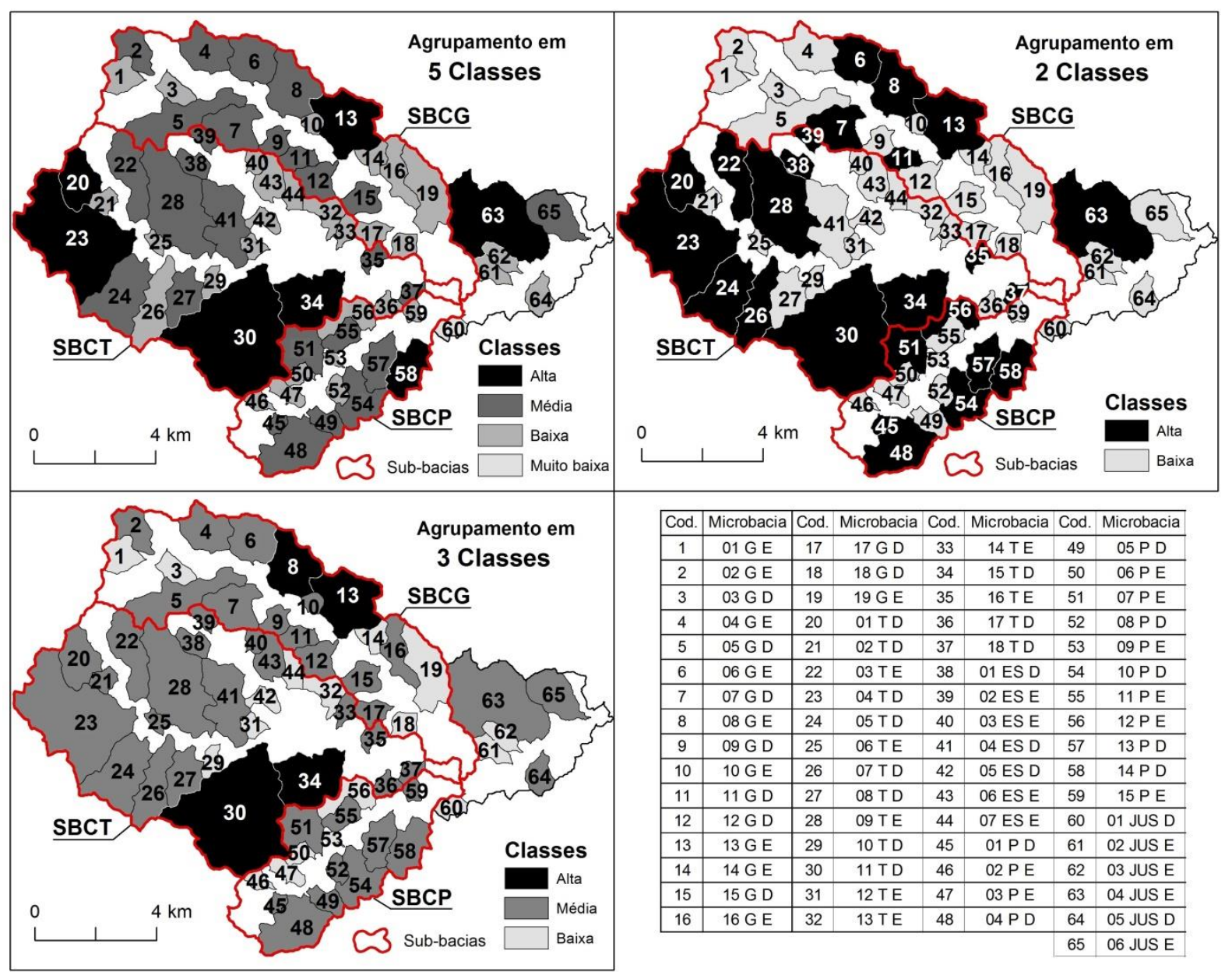

Figura 3. Suscetibilidade à inundação nas microbacias da BHRES, classificando-as em duas, três e cinco classes de vulnerabilidade.

Na classificação utilizando-se cinco classes, uma foi classificada como de muito baixa (01JUSD), 30 como de baixa, 27 como de média e sete como de alta suscetibilidade. Na SBCG, oito foram classificadas como de baixa, 10 como de média e uma de alta (13GE); na SBCT, doze foram classificados como de baixa, nove como de média e quatro como de alta; na SBCP, sete de baixa, sete de média e uma de alta (14PD); na área de drenagem à jusante das sub-bacias, uma foi classificada como de muito baixa (01JUSE), três foram classificadas como de baixa, uma como de média (06JUSE) e uma como de alta (04JUSE) suscetibilidade à inundação (Figura 3).

Independentemente do número de classes adotadas observa-se que o maior percentual e área de microbacias altamente suscetível à inundação concentram-se na SBCT. As microbacias localizadas na SBCP só apresentam alto percentual de área altamente vulnerável (74\%) quando são utilizadas apenas duas classes de suscetibilidade, pois com três classes, o percentual altamente vulnerável é zero e para cinco classes é 11,7\% (Figura 3).

Esta categorização, variável conforme as quantidades de classes adotadas, observada na avaliação das microbacias, tendeu a variar entre média e baixa, média e alta ou muito baixa e baixa, contudo, observaram-se duas microbacias onde a classificação foi antagônica. Estes foram os casos da microbacia 07TD, que foi classificada como baixa, com cinco classes, média, com três classes, e alta com duas classes; e a microbacia 12PE, que foi classificada como baixa com cinco e três classes e alta com duas classes. Sendo assim, o número de classes resultou em evidente variação dos resultados obtidos. 


\subsection{Identificação da similaridade morfométrica das microbacias}

Os três critérios de validação de clusters utilizados indicaram que, para as características morfométricas avaliadas, as microbacias ficariam melhor distribuídas se fossem divididas em dois grupos. Os grupos encontrados com a técnica de agrupamento "K-means", indicaram um grupo com 47 microbacias e outro com 18, enquanto o agrupamento obtido com a técnica FCM indicou um grupo com 44 e outro com 21 elementos, como apresentado na Figura 4.

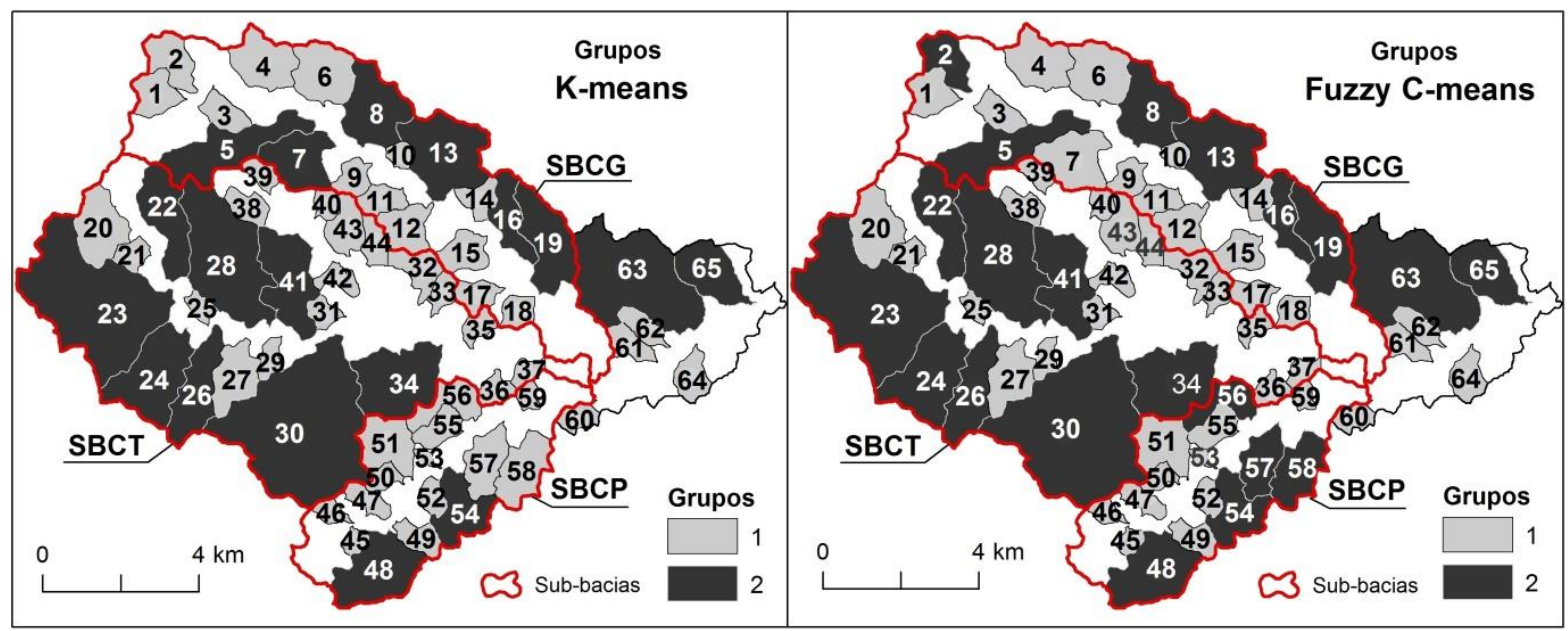

\begin{tabular}{|c|c|c|c|c|c|c|c|c|c|c|c|c|c|c|c|c|c|c|c|c|c|}
\hline Cod. & \begin{tabular}{|l|} 
Microbacia \\
\end{tabular} & Cod. & \begin{tabular}{|l|} 
Microbacia \\
\end{tabular} & Cod. & Microbacia & Cod. & Microbacia & Cod. & \begin{tabular}{|l|} 
Microbacia \\
\end{tabular} & Cod. & Microbacia & Cod. & Microbacia & Cod. & Microbacia & Cod. & Microbacia & Cod. & Microbacia & Cod. & Microbacia \\
\hline 1 & 01 GE & \begin{tabular}{|l|}
7 \\
\end{tabular} & 07 G D & 13 & $13 \mathrm{GE}$ & 19 & $19 \mathrm{GE}$ & 25 & \begin{tabular}{|l|}
$06 \mathrm{TE}$ \\
\end{tabular} & 31 & $12 \mathrm{TE}$ & 37 & 18 TD & 43 & 06 ES E & 49 & 05PD & 55 & $11 \mathrm{PE}$ & 61 & 02 JUS E \\
\hline 2 & $02 \mathrm{GE}$ & 8 & $08 \mathrm{GE}$ & 14 & $14 \mathrm{GE}$ & 20 & $01 \mathrm{TD}$ & 26 & 07 TD & 32 & $13 \mathrm{TE}$ & 38 & $01 \mathrm{ES} \mathrm{D}$ & 44 & $07 \mathrm{ESE}$ & 50 & $06 \mathrm{PE}$ & 56 & $12 \mathrm{PE}$ & 62 & 03 JUS E \\
\hline 3 & 03 GD & 9 & $09 \mathrm{GD}$ & 15 & $15 \mathrm{GD}$ & 21 & 02 TD & 27 & 08 TD & 33 & $14 \mathrm{TE}$ & 39 & $02 \mathrm{ESE}$ & 45 & $01 P D$ & 51 & $07 \mathrm{PE}$ & 57 & $13 P D$ & 63 & 04 JUS E \\
\hline 4 & $04 \mathrm{GE}$ & 10 & $10 \mathrm{GE}$ & 16 & $16 \mathrm{GE}$ & 22 & 03 TE & 28 & $09 \mathrm{TE}$ & 34 & 15 TD & 40 & $03 \mathrm{ESE}$ & 46 & $02 \mathrm{PE}$ & 52 & $08 P D$ & 58 & $14 \mathrm{PD}$ & 64 & 05 JUS D \\
\hline 5 & 05 GD & 11 & $11 \mathrm{GD}$ & 17 & $17 \mathrm{GD}$ & 23 & $04 \mathrm{TD}$ & 29 & $10 \mathrm{TD}$ & 35 & $16 \mathrm{TE}$ & 41 & 04 ES D & 47 & $03 \mathrm{PE}$ & 53 & $09 \mathrm{PE}$ & 59 & $15 \mathrm{PE}$ & 65 & 06 JUS E \\
\hline 6 & $06 \mathrm{GE}$ & 12 & $12 \mathrm{GD}$ & 18 & $18 \mathrm{GD}$ & 24 & $05 \mathrm{TD}$ & 30 & $11 \mathrm{TD}$ & 36 & $17 \mathrm{TD}$ & 42 & 05 ES D & 48 & 04 P D & 54 & $10 P D$ & 60 & 01 JUS D & & \\
\hline
\end{tabular}

Figura 4. Distribuição das microbacias da BHRES em grupos, utilizando-se as técnicas de agrupamento "Kmeans e Fuzzy C-means".

Confrontando-se as duas técnicas de agrupamento utilizadas (Figura 4), observa-se que ambas agruparam as microbacias de forma muito semelhante, apenas as microbacias 07GD, 12PE, 13PD e 14PD receberam classificações antagônicas.

Uma ferramenta de padronização a ser adotada, em análises de suscetibilidade com a utilização de intervalos de classe, seria o emprego de técnicas de validação de cluster para identificar, pelas características dos dados utilizados, o número de agrupamentos a serem adotados conforme a semelhança dos mesmos. No caso, as três técnicas de validação de cluster utilizadas apontaram para dois agrupamentos.

Comparando-se os grupos determinados pela análise de suscetibilidade utilizando-se duas classes, com os dois grupos gerados pela técnica de agrupamento "K-means", observa-se que das 47 microbacias agrupadas em um dos grupos, 35 foram classificadas como de baixa suscetibilidade à inundação. No outro grupo gerado pelo "K-means", das 18 microbacias agrupadas, 13 são classificadas como de alta suscetibilidade, ou seja, apesar de um dos grupos parecerem agrupar microbacias de baixa e outro de alta suscetibilidade à inundação, 17 microbacias $(26,15 \%)$ apresentaram-se em grupos diversos daqueles apontados pela metodologia de análise de suscetibilidade à inundação.

Os agrupamentos gerados pela metodologia FCM mostraram-se um pouco mais próximos que o "K-means", em relação aos resultados encontrados utilizando-se a análise de suscetibilidade, divergindo em $24,61 \%$ das microbacias. Das 44 microbacias agrupadas em um dos grupos pelo FCM, 34 foram classificadas na análise de suscetibilidade como de baixa e das 21 microbacias, agrupadas no outro grupo, 15 foram classificadas na análise de suscetibilidade como de alta. 
Comparando-se os dois grupos gerados pelas técnicas de agrupamento FCM e "Kmeans" observa-se que 6,15\% das microbacias foram agrupadas de forma distinta pelas duas técnicas.

Embora a metodologia FCM tenha se aproximado um pouco mais dos resultados obtidos por meio da análise de suscetibilidade que a "K-means", observa-se que ambas as técnicas de agrupamento divergiram da análise de suscetibilidade para algumas microbacias.

Como a metodologia para análise de suscetibilidade transforma os valores numéricos de cada parâmetro morfométrico em uma classe de suscetibilidade, classificando-os conforme sua implicação na inundação e ambas as metodologias de agrupamento utilizam os parâmetros brutos, a análise de suscetibilidade pareceu lançar luz sobre as implicações dos parâmetros na ocorrência de inundações. Apesar, de maneira geral, das microbacias com parâmetros semelhantes terem sido distribuídas dentro da mesma classe, a análise de suscetibilidade distinguiu-as, valorando as implicações com foco no objeto da análise.

\section{CONCLUSÕES}

A SBCT, seguida pela SBCP são as sub-bacias mais suscetíveis à inundação na BHRES. Contudo, uma análise mais pormenorizada permitiu localizar microbacias mais vulneráveis e destacar também a ocorrência de unidades hidrográficas que merecem atenção também na SBCG e na área de drenagem à jusante das sub-bacias.

A metodologia utilizada para identificação de áreas mais suscetíveis à inundação mostrou-se influenciável pela quantidade de classes de suscetibilidade adotada. Neste contexto, os critérios de validação de cluster parecem fornecer uma alternativa à definição da quantidade de classes, uma vez que, por meio deles, é possível inferir a quantidade de grupos similares que podem ser gerados a partir dos dados brutos.

As técnicas de agrupamento "K-means" e FCM, apesar de excelentes ferramentas para identificação de grupos de dados semelhantes, mostraram-se ferramentas auxiliares à classificação de áreas vulneráveis à inundação, enquanto a metodologia de análise de suscetibilidade pareceu ressaltar as diferenças nos parâmetros morfométricos, que implicam na suscetibilidade à inundação. Especificamente, neste estudo, a análise de suscetibilidade, com duas classes, como indicado pelos critérios de validação de cluster pareceu fornecer informações mais objetivas quanto à vulnerabilidade das microbacias.

\section{AGRADECIMENTOS}

Os autores agradecem à Fundação de Amparo à Pesquisa do Estado de Minas Gerais FAPEMIG pelo apoio financeiro a execução do projeto (Processo TEC - APQ-02118-12). Artigo derivado de parte da tese da primeira autora aprovada no Programa de Pós-graduação em Ecologia da Universidade Federal de Juiz de Fora.

\section{REFERÊNCIAS}

ALVES, J. M. P.; CASTRO, P. T. A. Influência de feições geológicas na morfologia da bacia do rio Tanque (MG) baseada no estudo de parâmetros morfométricos e análise de padrões de lineamentos. Revista Brasileira de Geociências, v. 33, n. 2, p. 117-127, 2003.

BELTRAME, Â. D. V. Diagnóstico do meio físico de bacias hidrográficas: modelo e aplicação. Florianópolis: Ed. da UFSC, 1994. 111 p.

CHRISTOFOLETTI, A. Geomorfologia fluvial. São Paulo: Edgard Blücher, 1981. 313 p. 
COOKE, R. U.; DOORNKAMP, J. C. Geomorphology in environmental management: an introduction. Oxford: Oxford University Press, 1974. 427 p.

DAVE, R. N. Validating fuzzy partition obtained through c-shells clustering. Pattern Recognition, v. 17, p. 613-623, 1996. http://dx.doi.org/10.1016/0167-8655(96)00026-8

FRITZSONS, E.; MANTOVANI, L. E.; RIZZI, N. E. Relação entre índices morfométricos de bacias hidrográficas e índices de integridade de floresta ciliar na bacia do Alto Capivari, Primeiro Planalto Paranaense. Colombo: Embrapa Florestas, 2009. 58 p.

HORTON, R. E. Erosional development of streams and their drainage basins; hidrophysical approach to quantitaive morphology. Geological Society of America Bulletin, v. 56, p. 807-813, 1945. http://dx.doi.org/10.1130/0016-7606(1945)56[275:EDOSAT]2.0.CO;2

INSTITUTO BRASILEIRO DE GEOGRAFIA E ESTATÍSTICA - IBGE. Ewbank da Câmara - MG, Folha SF-23-X-C-VI-2. Carta Topográfica. Escala: 1:50.000. Rio de Janeiro, 1980a.

INSTITUTO BRASILEIRO DE GEOGRAFIA E ESTATÍSTICA - IBGE. Juiz de Fora MG, Folha SF-23-X-D-IV-1. Carta Topográfica. Escala: 1:50.000. Rio de Janeiro, 1980b.

JENSON, S. K.; DOMINGUE, J. O. Extracting topographic structure from digital elevation data for geographic information system analysis. Photogrammetric Engineering and Remote Sensing, v. 54, n. 11, p. 1593-1600, 1988.

LIMA, W. D. P. A silvicultura e a água: ciência, dogmas e desafios. Rio de Janeiro: Instituto BioAtlântica, 2010. 54 p.

MACHADO, P. J. D. O. Diagnóstico ambiental e ordenamento territorial - instrumentos para a gestão da Bacia de Contribuição da Represa de Chapéu D’Uvas/MG. 2012. 243f. Tese (Doutorado em Geografia) - Universidade Federal Fluminense, Niterói, 2012.

MACHADO, W.; STIPP, N. A. F. Caracterização do manejo de solos na microbacia hidrográfica do Ribeirão dos apertados - PR. Geografia, v. 12, n. 2, p. 45-73, 2003.

MORISAWA, M. E. Quantitative geomorphology of some watersheds in the Appalachian Plateau. Geological Society of America Bulletin, v. 73, n. 9, p. 1025-1046, 1962. http://dx.doi.org/10.1130/0016-7606(1962)73[1025:QGOSWI]2.0.CO;2

NEVES NETO, C. D. C.; HESPANHOL, A. N. A atuação do estado brasileiro no processo de modernização agrícola e a incorporação do conceito de microbacias hidrográficas nas políticas públicas. Caderno Prudentino de Geografia, v. 31, n. 1, p. 94-109, 2009.

PAKHIRA, M. K.; BANDYOPADHYAY, S.; MAULIK, U. Validity index for crisp and fuzzy clusters. Pattern Recognition, v. 37, n. 3, p. 487-501, 2004. http://dx.doi.org/10.1016/j.patcog.2003.06.005

PINTO, V. G.; LIMA, R. N. de S.; RIBEIRO, C. B. de M.; MACHADO, P. J. de O. Diagnóstico físico-ambiental como subsídio a identificação de áreas vulneráveis à erosão na bacia hidrográfica do Ribeirão do Espírito Santo, Juiz de Fora (MG), Brasil. Revista Ambiente \& Água, v. 9, n. 4, p. 632-646, 2014 . http://dx. doi.org/10.4136/ambi-agua.1416 
RODRIGUES, W. A.; STARZYNSKI, R. Análise morfométrica da microbacia hidrográfica do córrego de Água Limpa. In: WORKSHOP EM MANEJO DE BACIAS HIDROGRÁFICAS, 2003, Botucatu. Proceedings... Botucatu: Faculdade de Ciências Agronômicas, UNESP, 2003. p. 144-163.

SANTOS, R. C. P. E. Avaliação de métodos baseados em sistemas fuzzy para mineração de dados georreferenciados. 2006. 87f. Dissertação (Mestrado em Engenharia Civil) Universidade Federal do Rio de Janeiro, Rio de Janeiro, 2006.

SINGH, V. K.; TIWARI, N.; GARG, S. Document Clustering using K-means, Heuristic Kmeans and Fuzzy C-means In: INTERNATIONAL CONFERENCE ON COMPUTATIONAL INTELLIGENCE AND COMMUNICATION SYSTEMS, 2011, Bali. Proceedings... Bali: IEEE, 2011. p. 297-301.

SOUZA, C. R. D. G. Suscetibilidade morfométrica de bacias de drenagem ao desenvolvimento de inundações em áreas costeiras. Revista Brasileira de Geomorfologia, v. 6, n. 1, p. 45-61, 2005.

STATSOFT INC. Statistica for Windows. Tulsa, 2002.

STRAHLER, A. N. Hypsometric (area-altitude) analysis of erosional topography. Geological Society of America Bulletin, v. 63, n. 11, p. 1117-1142, 1952. http://dx.doi.org/ 10.1130/0016-7606(1952)63[1117:HAAOET]2.0.CO;2

TEODORO, V. L. I.; TEIXEIRA D.; COSTA, D. J. L.; FULLER, B. B. O conceito de bacia hidrográfica e a importância da caracterização morfométrica para o entendimento da dinâmica ambiental local. Revista Uniara, v. 20, p. 137-156, 2007.

THE MATHWORKS INC. Matlab and Statistics Toolbox Release. Natick, 2012.

VILLELA, S. M.; MATTOS, A. Hidrologia aplicada. São Paulo: Mc Graw Hill, 1975. 245p.

WANG, D.; LAFFAN S. W.; LIU Y.; WU L. Morphometric characterisation of landform from DEMs. International Journal of Geographical Information Science, v. 24, n. 2, p. 305-326, 2010. http://dx.doi.org/10.1080/13658810802467969

XIE, X. L.; BENI, G. B. G. A Validity measure for Fuzzy Clustering. IEEE Transactions on Pattern Analysis and Machine Intelligence (PAMI), v. 13, n. 8, p. 841-847, 1991. http://dx.doi.org/10.1109/34.85677

ZÃVOIANU, I. Morphometry of drainage basins. 2. ed. [S.1.]: Elsevier, 1985. 250 p. 